


\title{
An Integrated Approach for Entry Mission Design and Flight Simulations
}

\author{
Ping $\mathrm{Lu}^{*}$ \\ Iowa State University \\ Ames, IA 50011-2271 \\ Prabhakara P. Rao ${ }^{\dagger}$ \\ Lockheed Martin Space and Strategic Missiles \\ Denver, CO 80201
}

\begin{abstract}
An integrated approach for entry trajectory design, guidance, and simulation is proposed. The key ingredients for this approach are an on-line 3 degree-offreedom entry trajectory planning algorithm and the entry guidance algorithm that generates the guidance gains automatically. When fully developed, such a tool could enable end-to-end entry mission design and simulations in 3DOF and 6DOF mode from de-orbit burn to the TAEM interface and beyond, all in one key stroke. Some preliminary examples of such a capability are presented in this paper that demonstrate the potential of this type of integrated environment.
\end{abstract}

\section{Introduction}

The traditional approach for entry mission planning and trajectory design is based on numerical optimization methods. The process is laborious and requires familiarity with the optimization software and experience with the optimization method because man-in-the-loop adjustments are inevitably needed. Consequently, trajectory analysis and design are typically restricted to baseline missions when only a limited amount of time is available. This process is also separated from the simulations needed to verify that the guidance and control systems can actually fly the designed trajectory. These verifications and validations happen only downstream when the reference trajectory data has been processed and translated into appropriate guidance system I-loads. A new approach based on a recently developed algorithm for entry trajectory planning and guidance has the potential to significantly shorten this timeconsuming process and allow quick turn-around in mission planning and trajectory design. ${ }^{1}$ The algorithm is originally developed with the objective of on-board applications. But the efficiency and versatility of the algorithm also make it ideal as the core of

\footnotetext{
*Professor, Department of Aerospace Engineering, Associate Fellow AIAA. Email: plu@iastate.edu

†Advanced Space Programs, Associate Fellow AIAA

Copyright (C) 2004 by the American Institute of Aeronautics and Astronautics, Inc. All rights reserved.
}

a tool that enables rapid entry trajectory design and mission planning with $3 \mathrm{DOF}$ or $6 \mathrm{DOF}$ simulations all at once. This algorithm has two parts. The first is a 3DOF entry trajectory planner that generates within 2-3 seconds on a desktop computer a feasible entry trajectory based on the vehicle model, current state, targeted final conditions and trajectory path constraints. The second is a self-tuned entry guidance law that automatically computes the guidance gains based on the reference trajectory generated by the trajectory planner. Therefore no post-processing and generation of guidance I-loads are required after the reference trajectory is found. The algorithm is readily implementable in a simulation environment where vehicle dynamics and other subsystems such as control, navigation and avionics are modeled. In this environment end-to-end simulations of entry fight can be performed with just one key stroke. Therefore many different possible mission scenarios involving different landing sites with various downrange and crossrange conditions can be investigated in trade studies in a relatively short period of time. The effects of vehicle parameters such as lift-to-drag ratio, angle of attack profile, and mass as well as trajectory constraints in peak heating rate or temperature, deceleration and dynamic pressure can be rapidly assessed. The information gathered in such studies may be passed back to the vehicle design 
team if necessary in the case of development of a new entry vehicle.

This paper will demonstrate some of the above mentioned capabilities of this technique. The mission scenarios are for an entry vehicle returning from the International Space Station orbit. The vehicle has a medium lift-to-drag ratio, and is to land at the Kennedy Space Center. The cases studies involve different entry conditions resulted from different deorbit opportunities, several nominal angle of attack profiles, and parametric variations in vehicle aerodynamic properties.

\section{Entry Trajectory Planning Algorithm}

The reference trajectory for the Space Shuttle is represented by a drag acceleration profile planned on the ground. ${ }^{2}$ By tracking this drag profile onboard, the longitudinal parameters of the entry trajectory (altitude, velocity, and range) are determined. The lateral motion is controlled by a bank reversal logic based on heading angle offset to the landing site. Different approaches have continued to be be investigated since the first flight of the Shuttle. ${ }^{1,3-8}$ The more recent efforts have focused on developing the capability of planning the reference trajectory onboard.

At the core of this fast approach for entry mission analysis and evaluation is a fast and reliable trajectory planning algorithm. Given initial conditions, landing site coordinates, terminal constraints, and trajectory path constraints, this algorithm will generate within 2-3 seconds a 3DOF feasible entry trajectory. This algorithm, implemented in a simulation environment, allow seamless integration of trajectory planning, guidance, control, and other subsystems in the simulations. This trajectory planning algorithm is described in detail in Ref. 1. For completeness and the convenience of the reader, this algorithm is briefly reviewed in this section.

\subsection{Entry Dynamics and Trajectory Con- straints}

The 3DOF point-mass dynamics of the entry vehicle over a spherical rotating Earth are described by following dimensionless equations of motion $^{9}$

$$
\begin{aligned}
\dot{r} & =V \sin \gamma \\
\dot{\theta} & =\frac{V \cos \gamma \sin \psi}{r \cos \phi} \\
\dot{\phi} & =\frac{V \cos \gamma \cos \psi}{r}
\end{aligned}
$$

$$
\begin{aligned}
\dot{V} & =-D-\left(\frac{\sin \gamma}{r^{2}}\right) \\
& +\Omega^{2} r \cos \phi(\sin \gamma \cos \phi-\cos \gamma \sin \phi \cos \psi) \\
\dot{\gamma} & =\frac{1}{V}\left[L \cos \sigma+\left(V^{2}-\frac{1}{r}\right)\left(\frac{\cos \gamma}{r}\right)\right. \\
& +2 \Omega V \cos \phi \sin \psi \\
& \left.+\Omega^{2} r \cos \phi(\cos \gamma \cos \phi-\sin \gamma \cos \psi \sin \phi)\right] \\
\dot{\psi} & =\frac{1}{V}\left[\frac{L \sin \sigma}{\cos \gamma}+\frac{V^{2}}{r} \cos \gamma \sin \psi \tan \phi\right. \\
& -2 \Omega V(\tan \gamma \cos \psi \cos \phi-\sin \phi) \\
& \left.+\frac{\Omega^{2}}{\cos \gamma} \sin \psi \sin \phi \cos \phi\right]
\end{aligned}
$$

where $r$ is the radial distance from the center of the Earth to the vehicle, normalized by the radius of the Earth $R_{0}=6378 \mathrm{~km}$. The longitude and latitude are 0 and $\phi$, respectively. The Earthrelative velocity $V$ is normalized by $V_{c}=\sqrt{g_{0} R_{0}}$ with $g_{0}=9.81 \mathrm{~m} / \mathrm{sec}^{2}$. The terms $D$ and $L$ are the aerodynamic drag and lift accelerations in $g$ 's, i. e., $D=\rho\left(V_{c} V\right)^{2} S_{r e f} C_{D} /\left(2 m g_{0}\right)$ and $L=$ $\rho\left(V_{c} V\right)^{2} S_{r e f} C_{L} /\left(2 m g_{0}\right)$, where $\rho$ is the atmospheric density, $S_{\text {ref }}$ the reference area of the vehicle, and $m$ the mass of the vehicle. Note that $D$ and $L$ are also functions of $\alpha$, the angle of attack, through the dependence of the drag and lift coefficients $C_{D}$ and $C_{L}$ on $\alpha$. The flight path angle of the Earthrelative velocity is $\gamma$ and $\sigma$ the bank angle. The velocity azimuth angle $\psi$ is measured from the North in a clockwise direction. The differentiation is with respect to the dimensionless time $\tau=t / \sqrt{R_{0} / g_{0}}$. Finally, $\Omega$ is the Earth self-rotation rate normalized by $\sqrt{g_{0} / R_{0}}$.

Typical inequality entry trajectory constraints include:

$$
\begin{gathered}
\dot{Q} \leq \dot{Q}_{\max } \\
|L \cos \alpha+D \sin \alpha| \leq n_{z_{\max }} \\
q \leq q_{\max } \\
{\left[\frac{1}{r}-V^{2}\right]\left(\frac{1}{r}\right)-L \cos \sigma_{E Q} \leq 0}
\end{gathered}
$$

where Eq. (7) is a constraint on the heating rate at a specified stagnation point on the surface of the vehicle, with $\dot{Q}=k \sqrt{\rho} V^{3.15}$ for a constant $k$. When applicable, this constraint may be replaced by a altitude-versus-velocity curve that represents the temperature limits at various body points. The constraint Eq. (8) is on the aerodynamic load (in $g$ 's) in the body-normal direction. Depending on the vehicle configuration and mission, this constraint may 
be replaced by the total load constraint:

$$
\sqrt{L^{2}+D^{2}} \leq n_{\max }
$$

Constraint Eq. (9) is on the dynamic pressure with $q=\rho\left(V_{c} V\right)^{2} / 2$. The parameters $\dot{Q}_{\max }$, $n_{z_{\max }}\left(n_{\max }\right)$, and $q_{\max }$ are vehicle-dependent constants. The constraints in Eqs. (7)-(9) are the "hard" constraints, meaning that they should be enforced strictly. When held in equality, the last constraint Eq.(10) is called the equilibrium glide condition (EGC) at $\sigma=\sigma_{E Q}$, where $\sigma_{E Q}$ is a specified constant. The EGC is obtained by omitting the Earth self-rotation terms and setting $\dot{\gamma}=0$ and $\gamma=0$ in Eq. (5). This constraint serves to reduce the phugoid oscillations in altitudes along the entry trajectory, and preserve bank angle margin so that $\sigma \geq \sigma_{E Q}$ during most of the flight. The latter allows sufficient trajectory control to account for dispersions. The constraint Eq. (10) is a soft constraint in the sense that its observance need not be absolutely strict, particularly in the final portion of the entry trajectory.

The entry trajectory terminates at a condition traditionally referred to as the Terminal Area Energy Management (TAEM) interface. This condition can be for instance a given value of velocity/Mach number. At the TAEM interface, the entry trajectory must have correct conditions to ensure successful TAEM flight and landing that will follow entry. These conditions form the terminal constraints on the entry trajectory in terms of:

$$
r_{f}=r_{T A E M}, V_{f}=V_{T A E M}, s_{f}=s_{T A E M}
$$

where $s_{f}$ is the value of range-to-go $s_{\text {togo }}$, defined to be the range from the vehicle to the tangency of the heading alignment cone (HAC) near an end of the runway for horizontal landing. The coordinates of the center of the HAC are known. In the case of other forms of landing and recovery (e.g., via parachute), HAC will represent the landing site although the term "HAC" is still used in this paper in a generic sense. The TAEM altitude $r_{T A E M}$, velocity $V_{T A E M}$ and range-to-HAC $s_{T A E M}$ are all specified for a given vehicle. In addition, the Earthrelative velocity vector at the TAEM interface should be pointing nearly to the HAC tangency. Let $\Delta \psi_{f}$ be the difference between the velocity azimuth angle and the line-of-sight angle from the vehicle to the HAC at the TAEM interface. This condition is then expressed by

$$
\left|\Delta \psi_{f}\right| \leq \Delta \psi_{T A E M}
$$

This condition stipulates that the final velocity vector should be directed at the HAC within a given tolerance $\Delta \psi_{T A E M}$.

The bank angle magnitude at the TAEM interface often times is also another parameter that is desired to be constrained. Too large a $\sigma_{f}$ could result in large transient response for TAEM guidance and control, and even make recovery unachievable for a less maneuverable entry vehicle. Thus, the constraint

$$
\left|\sigma_{f}\right| \leq \sigma_{T A E M}
$$

for a given $\sigma_{T A E M}>0$ may also be imposed.

\subsection{Trajectory Planning Problem and Algo- rithm Outline}

The entry reference trajectory generation problem is defined as follows: given the entry conditions, the path constraints and terminal conditions at the TAEM interface as in the preceding section, find the state history of $\mathbf{x}=\{r \theta \phi V \gamma \psi\}^{T}$ and the corresponding trajectory control $\mathbf{u}=\left\{\begin{array}{ll}\sigma & \alpha\end{array}\right\}^{T}$ such that:the state and control profiles $\mathbf{x}(t)$ and $\mathbf{u}(t)$ satisfy the 3DOF equations of motion Eqs. (1-6), all the required TAEM interface conditions, and the imposed trajectory path constraints. A pair $[\mathbf{x}(t) \mathbf{u}(t)]$ that meets the above conditions is called a feasible trajectory. We are concerned with only lifting entry flight, i.e., $L / D \neq 0$. Furthermore, we will assume that a nominal angle of attack profile is also specified for a given vehicle as it is in many cases. Such an $\alpha$-profile usually is determined on the basis of flight trim conditions and thermal considerations.

The trajectory planning algorithm developed in Ref. 1 tackles the problem in two major sequential steps: first determining the longitudinal profiles of the trajectory, and then completing the lateral profiles. In each step there is one single-parameter iteration involved. All the trajectory constraints except for Eq. (13) are enforced by the longitudinal profiles. The condition (13) is met by finding the point for the last bank reversal maneuver, and the equations of motion (1-6) are numerically integrated in the second step.

In the first step, the whole entry trajectory is divided into three phases. Each phase has its distinctive physical characteristics and the algorithm uses different approach in different phases:

1. Initial descent phase: the flight in this phase is a "controlled fall" in which the vehicle uses a constant bank angle, and the trajectory takes the 
vehicle from the entry interface at about $120 \mathrm{~km}$ in altitude to an altitude at about $80 \mathrm{~km}$. This is where the dynamic pressure has reached a sufficient level for aerodynamic lift to become influential in shaping the trajectory. The magnitude of the appropriate constant bank angle is determined by increasing the bank angle from zero at a fixed incremental and numerically integrating the equations of motion. The incremental process is stopped when the condition at the end of the initial descent satisfies a smooth transition into the next phase of quasi-equilibrium glide.

2. Quasi-equilibrium glide (QEG) phase: The QEG phase covers the majority of the entry trajectory where all the inequality path constraints must be observed and the range achieved must be correct for the vehicle to reach the landing site. In this phase the flight path angle $\gamma$ is small and varies relatively slowly. Setting $\cos \gamma=1$ and $\dot{\gamma}=0$ in Eq. (5) and ignoring Earth rotation gives

$$
L \cos \sigma+\left(V^{2}-\frac{1}{r}\right) \frac{1}{r}=0
$$

This relationship is called the quasi-equilibrium glide condition (QEGC). It is valid in lifting entry flight from an altitude of about 75-80 $\mathrm{km}$ to certain supersonic velocity which is dependent on the lifting capability of the vehicle. The QEGC is used in the trajectory planning algorithm as a motion integral. It allows conveniently the bank angle to be tied directly to the longitudinal trajectory state variables velocity and altitude. With the aid of the QEGC, the satisfaction of the requirements on range, velocity and altitude and observation of the inequality trajectory constraints are all accomplished by the iterative determination of a bank angle parameter (typically the value of bank angle at the midway point along the QEG phase).

3. Pre-TAEM phase : In this phase the velocity has reduced to a level close to the TAEM velocity, and the flight path angle as well as the altitude starts to decrease more rapidly. The QEGC is no longer valid. The algorithm numerically integrates the equations of motion backward from the given TAEM conditions to a selected preTAEM velocity (usually around Mach 5 ) where the QEGC phase connects with the pre-TAEM phase. The bank angle is defined by flying an altitude profile as a polynomial in velocity. No iteration is involved in this phase. In terms of execution sequence, the pre-TAEM phase is planned before the QEG phase so that the preTAEM results establish well defined conditions at the end of the reverse integration where the QEG phase ends. These conditions are then used in determining the bank angle profile in the QEG phase.

In the second major step, the longitudinal profiles (range, altitude, and flight path angle versus energy) found in the first step are tracked with linearized time-varying feedback control laws in the magnitude of the bank angle and angle of attack when angle of attack modulation is allowed. The sign of the bank angle is initially such that the heading error to the HAC is reduced. If the sign of the bank angle remains unchanged, the trajectory would overcorrect itself and miss the landing site. Therefore the sign is reversed at an appropriate point so that when the vehicle reaches the TAEM interface, the condition Eq. (13) is satisfied. The determination of the correct bank reversal point is the second oneparameter iteration in the algorithm, and involves numerically integrating the full equations of motion with the bank angle and angle of attack from the tracking laws. Once this second search is finished, a complete 3DOF feasible entry trajectory is found, and ready to be used as the reference trajectory for entry guidance.

\section{Entry Guidance Algorithm}

The entry guidance law used in this paper is based on receding-horizon control (RHC) approach. Consider the linearized dynamics of the system Eqs. $(1-6)$ about the reference trajectory

$$
\dot{\delta} \mathbf{x}=A(t) \delta \mathbf{x}+B(t) \delta \mathbf{u}
$$

where $\delta \mathbf{x} \in R^{6}$ is the state dispersion, $\delta \mathbf{u}=(\delta \sigma \delta \alpha)^{T}$ the control dispersion, and $A(\cdot): R \rightarrow R^{6 \times 6}$ and $B(\cdot): R \rightarrow R^{6 \times 2}$ are the Jacobians of the system Eqs. (1-6) with respect to the state and control vector, respectively. These two matrices are timevarying because the reference trajectory is timevarying. The regulation/stabilization problem seeks to find a control law $\delta \mathbf{u}=K(t) \delta \mathbf{x}$ to drive $\delta \mathbf{x} \rightarrow 0$ for any initial nonzero condition $\delta \mathbf{x}\left(t_{0}\right)$. The recedinghorizon control problem is defined to be an optimal control problem in which the performance index at any $t$

$$
J=\int_{t}^{t+T}\left[\delta \mathbf{x}^{T}(\tau) Q \delta \mathbf{x}(\tau)+\delta \mathbf{u}^{T}(\tau) R \delta \mathbf{u}(\tau)\right] d \tau
$$

is minimized for some $Q \geq 0$ and $R>0$, subject to system dynamics (16), initial condition $\delta \mathbf{x}(t)$, and 
the terminal constraint

$$
\delta \mathbf{x}(t+T)=0
$$

where $0 \leq T<\infty$ is a constant that defines the control horizon of the problem. The idea is to solve this optimal control problem in the finite moving horizon $[t, t+T]$ with the current state $x(t)$ as the initial condition. Let $\delta \mathbf{u}_{\text {optm }}(\cdot)$ be the optimal control obtained in $[t, t+T]$. Only the first data $\delta \mathbf{u}_{o p t m}(t)$ is used to be the current applied control $\delta \mathbf{u}(t)=\delta \mathbf{u}_{\text {optm }}(t)$. The rest of $\delta \mathbf{u}_{\text {optm }}(\cdot)$ is discarded. The process is then repeated for the next $t$. Because the control $\delta \mathbf{u}(\cdot)$ applied at any $t$ is dependent on the condition $\delta \mathbf{x}(t)$, the receding-horizon control strategy produces a feedback control.

A closed-form approximation to the recedinghorizon control law has been developed. ${ }^{10}$ Consider the above receding-horizon problem in the interval $[t, t+T]$. Divide this interval into $N$ subintervals of equal length $h=T / N$ for some integer $N \geq 3$. With the current state $\delta \mathbf{x}(t)$ known, a first-order prediction of $\delta \mathbf{x}(t+h)$ as a function of $\delta \mathbf{u}(t)$ is given by a Taylor series expansion at $t$

$$
\begin{aligned}
\delta \mathbf{x}(t+h) & \approx \delta \mathbf{x}(t)+h[A(t) \delta \mathbf{x}(t)+B(t) \delta \mathbf{u}(t)] \\
& =(I+h A) \delta \mathbf{x}+h B \delta \mathbf{u}
\end{aligned}
$$

Denote $A_{k}=A(t+k h), B_{k}=B(t+k h), \delta \mathbf{x}_{k}=$ $\delta \mathbf{x}(t+k h)$ and $\delta \mathbf{u}_{k}=\delta \mathbf{u}(t+k h), k=1, \ldots, N$. Then another first-order Taylor series expansion at $t+h$, together with Eq. (19), gives

$$
\begin{aligned}
\delta \mathbf{x}(t+2 h) & \approx \delta \mathbf{x}_{1}+h\left[A_{1} \delta \mathbf{x}_{1}+B_{1} \delta \mathbf{u}_{1}\right] \\
& \approx\left(I+h A_{1}\right)(I+h A) \delta \mathbf{x} \\
& +h\left(I+h A_{1}\right) B \delta \mathbf{u}+h B_{1} \delta \mathbf{u}_{1}(20)
\end{aligned}
$$

Continuing this process, we have

$$
\delta \mathbf{x}_{k} \approx \Delta_{k} \delta \mathbf{x}+\sum_{i=0}^{k-1} G_{k, i} \delta \mathbf{u}_{i}, \quad k=1, \ldots N
$$

where

$$
\begin{aligned}
\Delta_{k}= & \left(I+h A_{k-1}\right) \Delta_{k-1}, \quad \text { with } \Delta_{0}=I \\
G_{k, i}= & \left(I+h A_{k-1}\right) G_{k-1, i}, i=0,1, \ldots, k-2 \\
& \text { and } G_{k, k-1}=h B_{k-1}
\end{aligned}
$$

The subscript 0 in above expressions denotes the values at $t$. Let $L_{0}=\delta \mathbf{x}^{T}(t) Q \delta \mathbf{x}(t)+\delta \mathbf{u}^{T}(t) R \delta \mathbf{u}(t)$ and $L_{k}=\delta \mathbf{x}_{k}^{T} Q \delta \mathbf{x}_{k}+\delta \mathbf{u}_{k}^{T} R \delta \mathbf{u}_{k}, k=1, \ldots, N$. The integral in Eq. (17) is approximated by the standard trapezoidal formula for integrals:

$$
J \approx h\left(0.5 L_{0}+L_{1}+\ldots+L_{N-1}+0.5 L_{N}\right)
$$

Define an (2N)-dimensional vector $v=$ $\operatorname{col}\left\{\delta \mathbf{u}(t), \delta \mathbf{u}_{1}, \ldots, \delta \mathbf{u}_{N-1}\right\}$. If the $\delta \mathbf{x}_{k}$ 's in Eq. (24) are replaced by the prediction (21), the performance index is thus approximated by a quadratic function of $v$ :

$$
\begin{aligned}
\bar{J} & =\frac{1}{2} v^{T} H(t, N, h) v+\delta \mathbf{x}^{T} S(t, N, h) v \\
& +q\left(\delta \mathbf{x}, t, N, h, \delta \mathbf{u}_{N}\right)
\end{aligned}
$$

where $H \in R^{2 N \times 2 N}$ is positive definite for any $t \geq t_{0}$, integer $N$ and $h>0, S \in R^{6 \times 2 N}$ and $q$ is quadratic in $\delta \mathbf{x}$ and $\delta \mathbf{u}_{N}$. These terms are obtained directly by rearranging the expression of $J$ in Eq. (24) once $\delta \mathbf{x}_{k}$ 's are replaced by Eq. (21). Examples of the expressions of $H$ and $S$ for several values of $N$ can be found in Ref. 10. The constraint Eq. (18) can be approximated by setting $\delta \mathbf{x}_{N}=0$ from Eq. (21), which can be rewritten in a compact form

$$
M^{T}(t, N, h) v=-\Delta_{N} \delta \mathbf{x}
$$

where

$$
M^{T}=\left[\begin{array}{lll}
G_{N, 0} & \ldots G_{N, N-1}
\end{array}\right] \in R^{6 \times 2 N}
$$

The minimization of $\bar{J}$ in Eq. (25) with respect to $v$ subject to constraint (26) constitutes a quadratic programming (QP) problem. When $M^{T}$ has full rank, the above QP problem has a unique solution

$$
\begin{aligned}
v & =-\left\{\left[H^{-1}-H^{-1} M\left(M^{T} H^{-1} M\right)^{-1} M^{T}\right.\right. \\
& \left.\left.\times \quad H^{-1}\right] S^{T}+\left[H^{-1} M\left(M^{T} H^{-1} M\right)^{-1}\right] \Delta_{N}\right\} \delta \mathbf{x}
\end{aligned}
$$

Define an $2 \times 2 N$ matrix

$$
I_{2 N}=\left\{I_{2 \times 2}, 0, \ldots .0\right\}
$$

A closed-form, linear, time-varying feedback control law for $\delta \mathbf{u}(t)$, denoted by $\delta \mathbf{u}(t ; N, h)$ to signify its dependence on time and the values of $N$ and $h$, is then obtained from the first two equations in Eq. (28)

$$
\delta \mathbf{u}(t ; N, h)=I_{2 N} v \triangleq K(t, N, h) \delta \mathbf{x}(t)
$$

The guidance law Eq. (30) regulates the trajectory in both longitudinal and lateral directions to follow the reference trajectory. All the feedback gains are generated automatically from the vehicle model and reference trajectory as outlined above. Therefore there is no need for a separate bank reversal logic in the entry guidance algorithm as it is in the Shuttle entry guidance. This is particularly convenient during the development and analysis phase when the vehicle design is evolving, and different mission scenarios are examined. No extensive guidance logic 
re-design and adjustments are required for each new vehicle design update and changing mission profile. However, this approach has one possible drawback in the operational phase when significant aerodynamic modeling uncertainty exists. In the presence of large aerodynamic modeling mismatch, it might become difficult for the vehicle to follow closely both the longitudinal and lateral profiles of the reference trajectory which is designed on the basis of the nominal aerodynamic model. In such a case a guidance law may be more robust that only flies the reference longitudinal profiles, and controls the heading of the vehicle by a simple bank reversal logic. ${ }^{6}$

\section{Applications}

To demonstrate the application of the the above described approach, entry missions from the International Space Station (ISS) orbit for landing at the Kennedy Space Center (KSC) are considered. The entry vehicle has a medium lift-to-drag ratio. The TAEM interface is defined at a relative velocity of Mach 3. The following TAEM conditions are imposed:

$$
\begin{aligned}
& \text { altitude }=24.384 \mathrm{~km}(80,000 \mathrm{ft}) \\
& \text { range-to-HAC }=55.56 \mathrm{~km}(30 \mathrm{~nm}) \\
& \text { heading-to-HAC offset } \leq 5 \mathrm{deg}
\end{aligned}
$$

In addition, the trajectory is further constrained by

$$
\begin{array}{r}
\dot{Q} \leq 1134893 \mathrm{~W} / \mathrm{m}^{2}\left(100 \mathrm{BTU} / \mathrm{ft}^{2}-\mathrm{sec}\right) \\
\sqrt{L^{2}+D^{2}} \leq 2.5 \mathrm{~g} \\
q \leq 14364 \mathrm{~N} / \mathrm{m}^{2}(300 \mathrm{psf})
\end{array}
$$

The simulation environment is a tool developed at the NASA Marshall Space Flight Center, called Marshall Aerospace VEhicle Representation In C II (MAVERIC II). This is a modularized high-fidelity simulation software that allows the user to use either built-in sub-system models or replace them with different vehicle model, sub-systems and algorithms. The mean and dispersed wind data from the Global Reference Atmospheric Model (GRAM) ${ }^{11}$ is available in MAVERIC II for nominal and dispersed simulations. The entry guidance algorithms described in the preceding sections are implemented in MAVERIC II for the vehicle used in this paper. All the simulations are performed in MAVERIC II. At this point the control system design is not yet implemented for this vehicle, thus all the simulations are 3DOF simulations.

\subsection{Entry Flight Assessment for Multiple De- Orbit Options}

The objective of this type of analysis is to assess the capability of the vehicle to accommodate a range of de-orbit opportunities and still ensure a successful entry flight toward the primary landing site. The orbit from which the entry vehicle returns is assumed to be an orbit with the same altitude and inclination as those of the ISS orbit. Six de-orbit opportunities are examined here that result in different entry conditions at the entry interface defined at the altitude of $121.92 \mathrm{~km}(400,000 \mathrm{ft})$. These cases correspond to tests 13 to 17 in Ref. 12 . The entry conditions are summarized in Table 1. They are separated into two groups, Mission A1 - Mission A3, and Mission B1 Mission B3. The defining difference between the two groups is in their entry flight path angles: group A has a shallower entry night path angle thus longer downranges, whereas group B has a steeper entry flight path angle and shorter downranges as a result. In each group, the first mission (A1 or B1) corresponds to de-orbit at a best opportunity for landing at the KSC. Therefore the crossrange is minimum. The other two missions in each group are for the cases when the de-orbit maneuver takes place one orbit earlier and one orbit later than the best opportunity, respectively. The consequence is a large right crossrange or a large left crossrange at the entry interface. The negative sign for crossrange in Table 1 indicates a left crossrange.

The integrated approach described in this paper enables an end-to-end simulation for each of the possible de-orbit opportunity without the need to wait for off-line generation of a reference entry trajectory for a particular set of entry conditions. Instead, the reference entry trajectory is planned in the first entry guidance cycle. If the entry conditions are such that a feasible reference trajectory cannot be found for the vehicle, the trajectory planner sends back a flag to so indicate and provide a possible reason why a trajectory does not exist. The de-orbit maneuver then should be adjusted accordingly.

All the six set of entry conditions are feasible for this entry vehicle. Figure 1 shows the ground tracks of the 6 missions. Figures 2 and 3 illustrate the comparisons of altitude-vs-velocity, bank angle and angle of attack histories of the reference trajectory and the simulated trajectory for Mission A1. The closedloop guided trajectory follows the reference trajectory quite well in most of fight. The differences in initial portion of the trajectory can be attributed to the differences between the high-fidelity simulation 
environment (including winds) and the relatively less extensive modeling used in the trajectory planning algorithm for efficiency. The same comparisons for Mission A2 and A3 are included in Figs. 4 and 5 where the similar observations can be made. Figure 5 exhibits close symmetry in bank angle histories for Mission A2 and A3, which is to be expected, given that the entry conditions for these missions are nearly symmetric. This result also demonstrates the desired predictability of the outcome of the trajectory planning algorithm.

Table 2 summarizes the TAEM condition precision of all the 6 missions from MAVERIC II simulations. The precision is easily at the best possible level as far as the performance of any type of entry guidance is concerned.

\subsection{Flying Different $\alpha$-Profiles}

It is well known that the angle-of-attack profile flown by the entry vehicle affects considerably the footprint of the entry trajectories. Perhaps somewhat less well known is the fact that different $\alpha$ profiles also have different predictable impact on the heat load absorbed by the vehicle. In their classic work Allen and Eggers show that the total heat load $Q$ can be approximated by ${ }^{13}$

$$
Q \approx \frac{1}{4} m V_{0}^{2}\left(\frac{C_{f} S_{w}}{C_{D} S_{\text {ref }}}\right)
$$

where $C_{f}$ is the equivalent skin-friction coefficient, $S_{w}$ the wetted area, and $V_{0}$ the entry velocity. Different $\alpha$-profiles will result in difference values for $C_{D}$, hence different heat load, assuming that the variation in $C_{D}$ does not lead to the variation in $C_{f}$. Higher $\alpha$ will produce higher $C_{D}$, and thus lower heat load, and vice versa. If the flight times do not differ appreciably with different $\alpha$-profiles (as usually the case), the heat rate along the trajectory will also follow the same trend as the heat load decreases or increases when the $\alpha$-profile is changed. Possible restricting factors that may limit the option of flying different $\alpha$-profiles include trim requirement in flight control and thermal protection considerations.

The change of the nominal $\alpha$-profile will alter the entry trajectory, and a new reference trajectory has to be planned in a traditional analysis environment. The integrated approach presented in this paper readily handles the need to assess the effects of flying different $\alpha$-profiles without any additional preparation work. The nominal $\alpha$-profile is an optional input to the software, and the simulation is ready to go as soon as the $\alpha$-profile is selected. As a demon- stration, a lower $\alpha$-profile and a higher $\alpha$-profile are used in addition to the nominal $\alpha$-profile shown in Figs. 3 and 5. The difference is a $\pm 5 \mathrm{deg}$ in $\alpha$ at hypersonic speeds. The peak heat rate limit for the lower $\alpha$-profile is increased to $1,191,637.7 \mathrm{~W} / \mathrm{m}^{2}(105$ $\mathrm{BTU} / \mathrm{ft}^{2}$-sec $)$, and decreased to $907,914.4 \mathrm{~W} / \mathrm{m}^{2}(80$ $\mathrm{BTU} / \mathrm{ft}^{2}$-sec) for the higher $\alpha$-profile. All the other conditions/constraints remain the same as in Mission A1.

Figure 6 shows the variations of bank angles and the $3 \alpha$-profiles from the MAVERIC II simulations. The $\alpha$-profile labeled as "medium" is the one used in Figs. 1-5 earlier. The trajectories in the velocityaltitude space are plotted in Fig. 7. The effects of $\alpha$-profile at hypsersonic speeds are visible in Fig. 7: the trajectory is higher with a higher $\alpha$-profile and lower with a lower $\alpha$-profile. The impact on the heat rates is more evident in Fig. 8. Even with the increased peak heat rate limit, the trajectory with the lower $\alpha$-profile still rides on the heat rate constraint boundary in an extended period. On the other hand, with a higher $\alpha$-profile, the trajectory can now easily accommodate a $20 \%$ reduction in the peak heat rate limit when compared to the baseline mission. The TAEM condition precision in all these cases are comparable to the precision seen in Table 2.

The change of $\alpha$ profile affects the lift and drag of the vehicle, therefore could change the feasibility of some missions. For instance, Mission A2 becomes infeasible for this vehicle when the lower $\alpha$-profile is flown, because the reduction in lift renders the crossrange in Mission A2 too large for the vehicle. On the other hand, all 3 missions B1-B3 are not achievable by the vehicle when the lower $\alpha$-profile is used, because the energy dissipates too slowly with the shorter downranges (cf. Table 1) and reduced drag deceleration.

\subsection{Variations in $C_{L}$ and $C_{D}$}

As the vehicle design evolves, the design modifications are bound to bring about changes in the aerodynamic properties of the vehicle. For lifting entry any increase in the lift-to-drag ratio will only increase the capability of the vehicle to shape its entry trajectory. It is the reductions in the lift-to-drag ratio that will cause the reduction in corssrange capability of the vehicle. Therefore the design impact on mission needs to be assessed as the design evolution progresses. The aerodynamic model is a user-supplied module to the entry trajectory planning and entry guidance software. Once it is provided, the entry guidance software is ready to be used in simulations 
Table 1 Entry conditions at the altitude of $121.92 \mathrm{~km}(400,000 \mathrm{ft})$

\begin{tabular}{ccccc}
\hline \hline Mission & $V_{0}(\mathrm{~m} / \mathrm{s})$ & downrange $(\mathrm{km})$ & crossrange $(\mathrm{km})$ & $\gamma_{0}(\mathrm{deg})$ \\
\hline A1 & 7625.0 & 8160 & 148 & -1.2492 \\
A2 & 7625.15 & 8715 & 883 & -1.2493 \\
A3 & 7625.72 & 8197 & -778 & -1.2490 \\
B1 & 7622.0 & 6519 & 59 & -1.4379 \\
B2 & 7621.3 & 6554 & 809 & -1.4380 \\
B3 & 7622.79 & 6589 & -848 & -1.4376 \\
\hline \hline
\end{tabular}

without further updates.

With the trajectory for Mission A1 in Section 4.1 as the baseline, two hypothetic cases are presented here to show the application of the proposed approach: in one case a uniform $20 \%$ reduction in the lift coefficient $C_{L}$ is assumed while $C_{D}$ remains the same; the other case has a constant $20 \%$ increase in $C_{D}$ with $C_{L}$ fixed at the baseline level. Note that these variations are not treated as modeling errors, and the trajectory planner and entry guidance algorithm are fed with the actual changes. Figure 9 illustrates the corresponding bank angle histories along the two trajectories plus the baseline bank angle for comparison. In both cases the bank angle is smaller compared to baseline, because the $L / D$ ratio is decreased. The trajectories in the velocity-altitude space are shown in Fig. 10. With the reduction in $C_{L}$, the trajectory is lower as expected. With the increase in $C_{D}$, the trajectory stays more aloft. The effects of the different altitudes at the same velocity on heat rate are clear in Fig. 11. Again, with the increase in $C_{D}$, the heat rate is decreased as discussed in Section 4.2. Combinations of simultaneous increase in $C_{D}$ and decrease in $C_{L}$ have also been examined. The qualitative trends are the same as seen here.

When the drag coefficient $C_{D}$ is increased by $20 \%$, this vehicle is still capable of flying all the six missions listed in Table 1 . But when $C_{L}$ is reduced by $20 \%$, the vehicle cannot fly the missions with large crossanges anymore (Mission A2, A3, B2, and B3).

\section{Conclusions}

The conventional methodology for entry mission design, guidance, simulation and verification follows a sequential "divide-and-conquer" approach. Recent development of on-line entry trajectory planning algorithm and entry guidance algorithm with automatic gains generation allows seamless integration of these processes in a single environment where trajectory design, guidance, control, and simulation are all performed with a key stroke. This paper presents some preliminary examples of such capability in a high-fidelity simulation environment. With the completion of development of models and algorithms for other sub-systems, such an analysis and simulation tool could significantly increase the effectiveness, efficiency and reduce the design and development cycle associated with entry missions.

\section{Acknowledgments}

This work has been supported in part by the NASA contract NAS8-01098. The assistance by Dr. Zuojun Shen with the entry trajectory planning software is greatly appreciated.

\section{References}

1 Shen, Z., and Lu, P., "On-Board Generation of Three-Dimensional Constrained Entry Trajectories", Journal of Guidance, Control, and Dynamics, Vol. 26, No. 1, 2003, pp. 111-121.

2 Harpold, J. C., and Graves, C. A., "Shuttle Entry Guidance," The Journal of the Astronautical Sciences, Vol. XXXVII, No. 3, 1979, pp. 239268.

3 Gamble, J. D., Cerimele, C. J., Moore, T. E., and Higgins, J., "Atmospheric Guidance Concepts for an Aeroassisted Experiment," The Journal of the Astronautical Sciences, Vol.36, No. 1-2, 1988, pp. 45-71.

4 Fuhry, D. P., "Adaptive Atmospheric Reentry Guidance for the Kistler K-1 Orbital Vehicle," Proceedings of AIAA Guidance, Navigation, and Control Conference, Vol. 2, pp. 12751288, 1999, AIAA, Reston, VA.

5 Zimmerman, C., Dukeman, G., and Hanson, J., "An Automated Method to Compute Orbital Entry Re-Entry Trajectories with Heating Constraints", Journal of Guidance, Control, and Dynamics, Vol. 26, No. 4, 2003, pp. 523-529. 
Table 2 TAEM interface condition errors from MAVERIC II simulations

\begin{tabular}{cccc}
\hline \hline Mission & altitude $\Delta r_{f}(\mathrm{~m})$ & range $\Delta s_{f}(\mathrm{~km})$ & heading error $\Delta \psi_{f}(\mathrm{deg})$ \\
\hline A1 & 96.86 & 1.124 & 0.72 \\
$\mathrm{~A} 2$ & 280.58 & 1.641 & 0.19 \\
$\mathrm{~A} 3$ & 362.62 & 0.893 & -3.02 \\
$\mathrm{~B} 1$ & -50.1 & 0.329 & 0.006 \\
$\mathrm{~B} 2$ & 257.85 & -3.706 & 2.85 \\
$\mathrm{~B} 3$ & 450.08 & 1.665 & 0.99 \\
\hline
\end{tabular}

6 Dukeman, G. A., "Profile-Following Entry Guidance Using Linear Quadratic Regulator Theory", AIAA Paper 2002-4457, Guidance, Navigation, and Control Conference, Monterey, CA, August 5-8, 2002.

7 Mease, K. D., Chen, D. T., Teufel, P., and Schoenenberger, H., "Reduced-Order Entry Trajectory Planning for Acceleration Guidance", Journal of Guidance, Control, and Dynamics, Vol. 25, No. 2, 2002, pp. 257-266.

8 Roenneke, A. J., "Adaptive On-board Guidance for Entry Vehicle," AIAA Paper 2001-4048, Guidance, Navigation, and Control Conference, Montreal, Canada, August 6-9, 2001.

9 Vinh, N. X., Busemann, A., and Culp, R. D., Hypersonic and Planetary Entry Flight Mechanics, The University of Michigan Press, Ann Arbor, MI, 1980, pp. 26-27

10 Lu, P., "Regulation about Time-Varying Trajectories: Precision Entry Guidance Illustrated," Journal of Guidance, Control, and Dynamics, Vol. 22, No. 6, 1999, pp. 784-790.

11 Justus, C. G. and Johnson, D. L., The NASA/MSFC Global Reference Atmospheric Model: 1999 Version (GRAM-99), NASA TM209630, May, 1999

12 Hanson, J., "Advanced Guidance and Control Methods for Reusable Launch Vehicles: Test Results," AIAA Paper 2002-4561, Guidance, Navigation, and Control Conference, Monterey, CA, August 5-8, 2002.

13 Allen, H. J., and Eggers, A. J., A Study of Motion and Aerodynamic Heating of Ballistic Missiles Entering the Earth's Atmosphere at High Supersonic Speeds, NACA Report 1381, 1958.

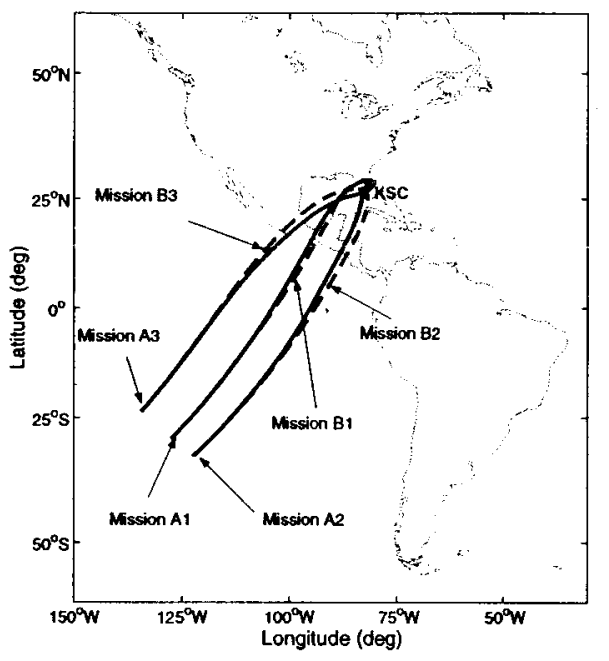

Fig. 1 Ground tracks of all 6 entry flight trajectories

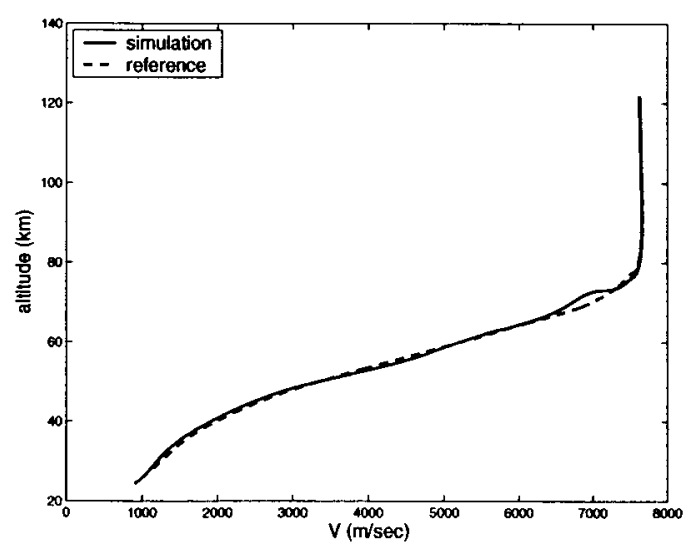

Fig. 2 Comparison of altitude and velocity for Mission A1 

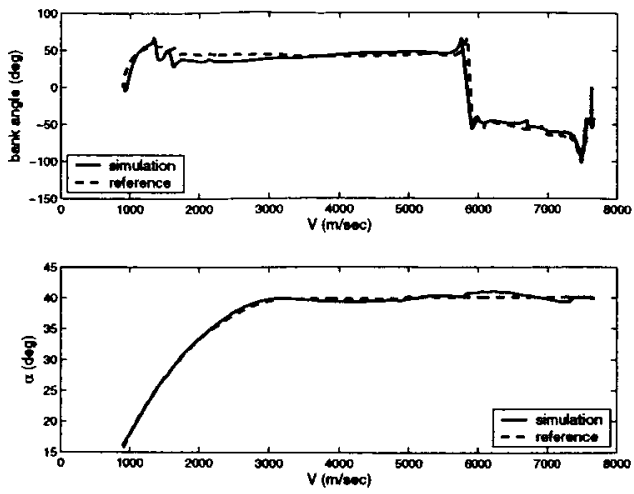

Fig. 3 Comparison of bank angle and $\alpha$ for Mission A1

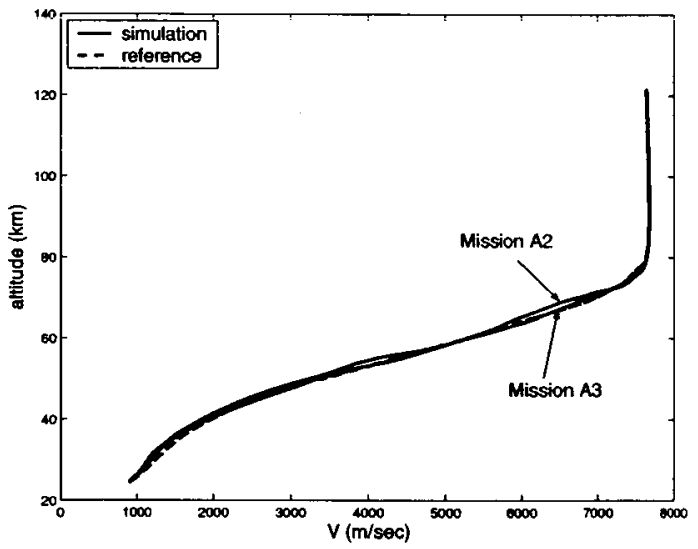

Fig. 4 Comparison of altitude and velocity for Mission A2 and A3
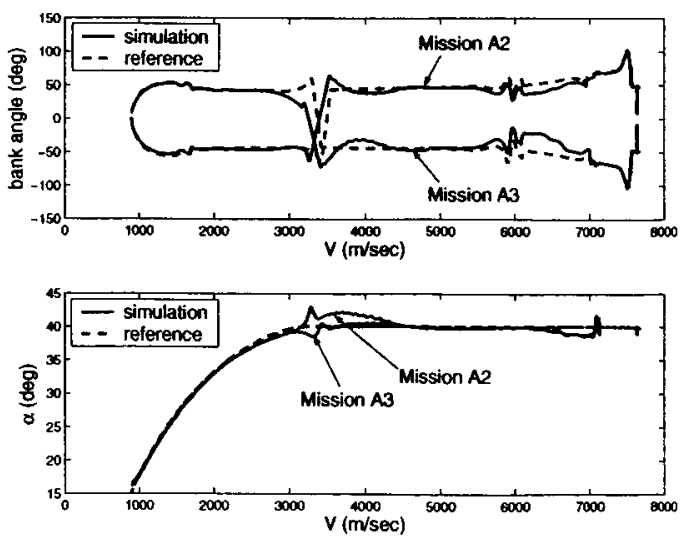

Fig. 5 Comparison of bank angle and $\alpha$ for Mission $\mathrm{A} 2$ and $\mathrm{A} 3$
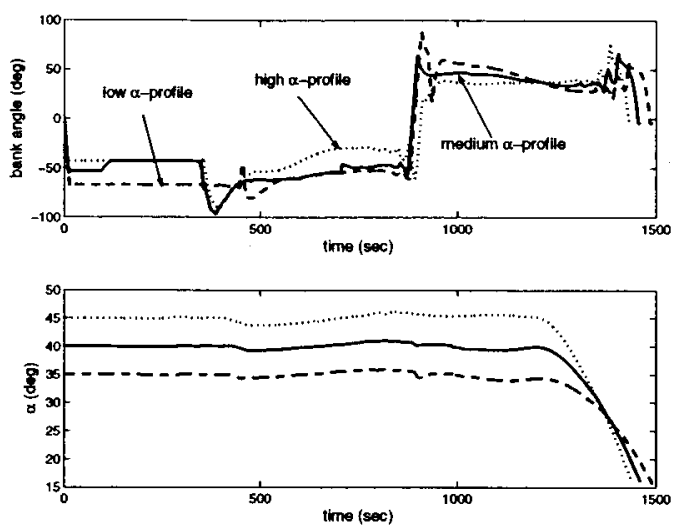

Fig. 6 Comparison of bank angles with different nominal $\alpha$-profiles for Mission A1

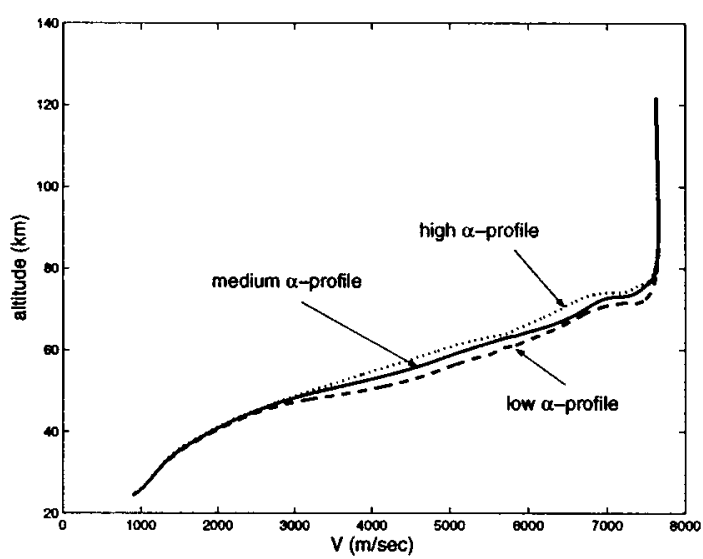

Fig. 7 Comparison of trajectories with different nominal $\alpha$-profiles for Mission A1

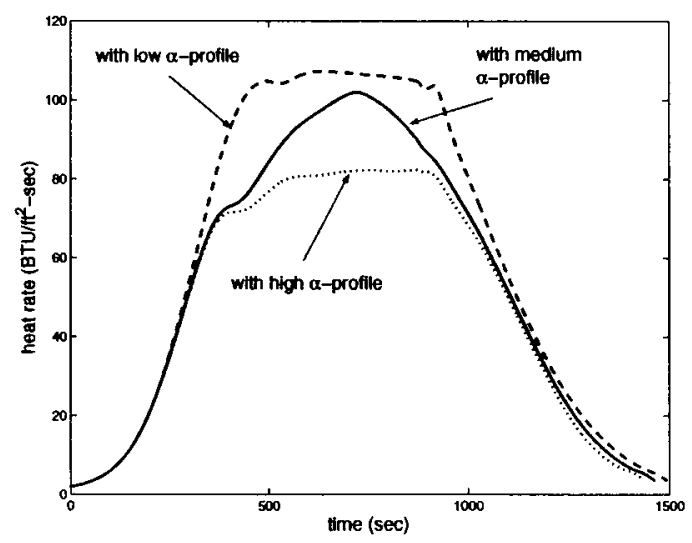

Fig. 8 Heat rates with different nominal $\alpha$ profiles for Mission A1 


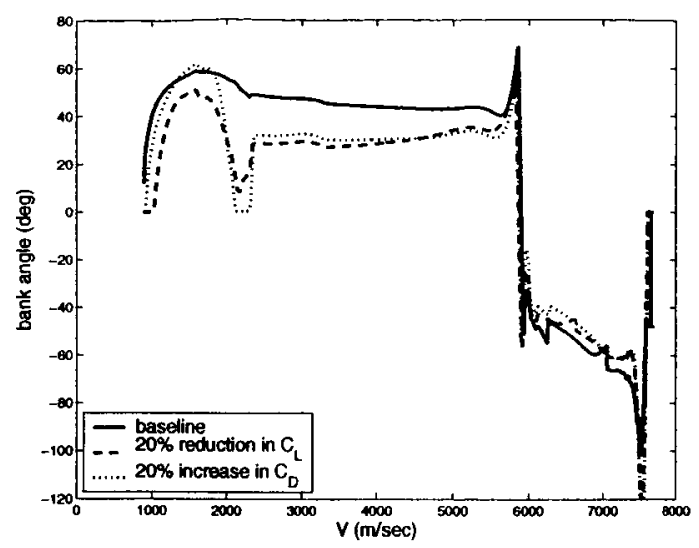

Fig. 9 Bank angles with variations in $C_{L}$ or $C_{D}$ for Mission A1

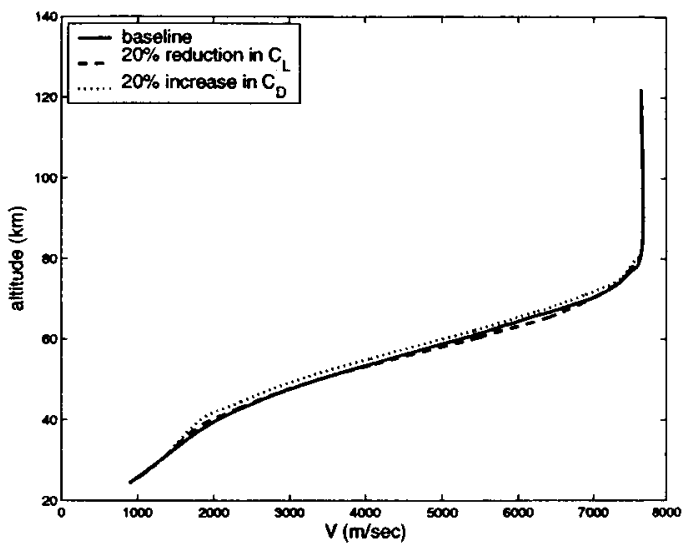

Fig. 10 Trajectories with variations in $C_{L}$ or $C_{D}$ for Mission A1

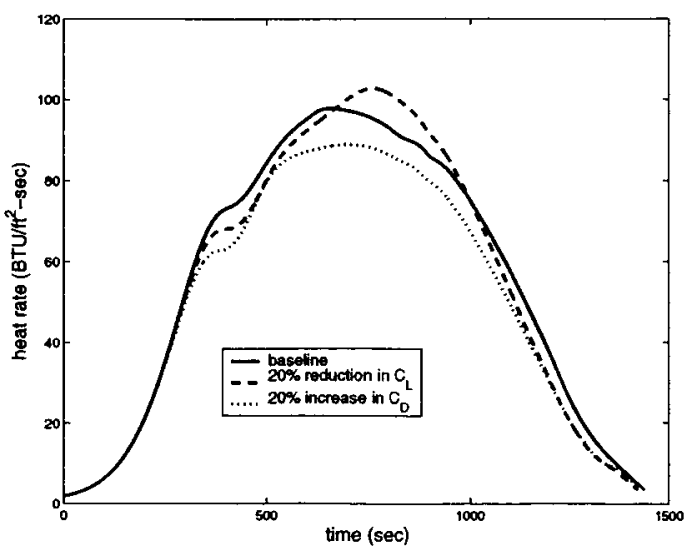

Fig. 11 Heat rates with variations in $C_{L}$ or $C_{D}$ for Mission A1 